\title{
Quantum mechanics/molecular mechanics methodology for metals based on orbital-free density functional theory
}

\author{
Xu Zhang and Gang Lu* \\ Department of Physics and Astronomy, California State University Northridge, Northridge, California 91330-8268, USA
}

(Received 9 September 2007; published 10 December 2007)

\begin{abstract}
We present a quantum mechanics (QM)/molecular mechanics (MM) formalism for coupling density functional theory (DFT) based quantum simulations to classical atomistic simulations for metals. The multiscale methodology is applicable to systems where important quantum phenomena are confined to a small region, but their impacts could be felt over much larger scales. The concurrent coupling between QM and MM regions is treated quantum mechanically via the orbital-free density functional theory (OFDFT). We propose two energetic formulations for the QM region: one is based on OFDFT and the other based on the Kohn-Sham (KS) DFT. In the first case, the degree of freedom is the electron charge density in the QM region, and the total energy functional is directly minimized with respect to the charge density. In the second case, the degrees of freedom are KS orbitals in the QM region. An embedding potential representing the influence of the larger MM region onto the QM region is included in the KS Hamiltonian for the QM region, which is solved selfconsistently. Calculations for a perfect lattice and vacancy clusters of aluminum demonstrate that the present QM/MM approaches yield excellent results both in terms of energetics and electron density.
\end{abstract}

DOI: 10.1103/PhysRevB.76.245111

PACS number(s): 71.15.Mb, 71.15.Dx, 61.72.Ji

\section{INTRODUCTION}

The challenge in simulations of materials science and engineering is that real materials usually exhibit phenomena at one scale, which require a very accurate and computationally expensive description, and phenomena at another scale, for which a coarser description is satisfactory and, in fact, necessary to avoid prohibitively large computations. ${ }^{1}$ For example, quantum mechanics $(\mathrm{QM})$ is required for a proper treatment of bond breaking, bondforming, charge transfer, electron excitation, magnetism, etc., in materials. However, due to the demanding computational cost, the application of QM is limited to relatively small systems consisting of up to a few hundreds of atoms. On the other hand, atomistic simulations based on empirical interatomic potentials are often capable of describing small-amplitude vibrations and torsions, elastic deformation and electrostatic interactions, etc., in many materials and biological systems. ${ }^{2}$ Termed as molecular mechanics (MM) methods, these empirical atomistic approaches can treat millions of atoms, with simpler potentials, even billions of atoms. ${ }^{3}$

Multiscale approaches that combine methods specialized at different scales, effectively distributing the computational power where it is needed most, are the answer to the challenge. In recent years, many concurrent multiscale approaches have been developed in materials simulations, across several length scales. ${ }^{4-19}$ In this paper, we focus on the so-called QM/MM coupling algorithms, ${ }^{2,14-17}$ which combine the accuracy of QM descriptions with the low computational cost of MM simulations to deal with large systems with desired accuracy.

QM/MM coupling schemes can be divided into two categories-mechanical coupling and quantum coupling, depending on how the interaction energy between QM and MM is formulated. With the mechanical coupling, the interaction energy between the QM region (region I) and MM region (region II) is calculated at the MM level. On the other hand, the interaction energy between regions I and II is calculated at the QM level in quantum coupling. Although the mechanical coupling is computationally much simpler than the quantum coupling, it has many drawbacks, the most important one being the electronic interaction of the two regions ignored. For example, in the mechanical coupling, the electrostatic and exchange-correlation interactions between the nuclei and the electrons in regions I and II are not considered explicitly. As a consequence, the electronic properties of region I are not accurately captured. Another drawback of the mechanical coupling, which is of practical importance, is the rather limited availability of empirical potentials for treating the coupling. ${ }^{2}$ In contrast, the quantum coupling is more accurate as it treats the interaction between the two regions quantum mechanically. Depending on the level of quantum descriptions, the extent of the electronic coupling is varied, from merely long-range electrostatic interaction to a full Coulomb interaction, including short-ranged exchange correlations.

In this paper, we present a $\mathrm{QM} / \mathrm{MM}$ coupling that is formulated based on the so-called orbital-free density functional theory (OFDFT). OFDFT is an efficient implementation of density functional theory (DFT) that approximates the kinetic energy of noninteracting electrons in terms of their density. ${ }^{20-22}$ We propose two methods for treating the primary subsystem or region I: one is based on OFDFT and the other based on Kohn-Sham (KS)-DFT. ${ }^{23}$ The first method follows closely the original work of Choly et al., ${ }^{11}$ but with significant improvement over the original method in terms of coupling accuracy. The second method is an important extension to the first method, which allows the treatment of much more complex systems than the first method could. As it will become clear later, the coupling in the first method is achieved via electron density, whereas in the second method, the coupling is realized in terms of KS orbitals. Both methods involve self-consistent determination of a single-particle embedding potential that represents the full quantum me- 
chanical effects of region II (including both nuclei and electrons) onto the electrons of region $\mathrm{I}^{2,10}$ The embedding potential is then included in the single-particle KS Hamiltonian of region I, for which a quantum mechanical problem is solved.

In Sec. II, we present the OFDFT formalism for treating the interaction energy, embedding potential and their contributions to the ionic force. The details of implementation of both methods are described In Sec. III. Various tests are performed to validate and contrast the coupling methods. In Sec. IV, we present an application of the first coupling method for calculating the binding energy of vacancy clusters in aluminum followed by a summary in Sec. V.

\section{QUANTUM COUPLING FORMALISM}

In general, the entire system of a QM/MM simulation is partitioned into two regions: region I treated by $\mathrm{QM}$ and region II treated by MM. In the present approaches, the interaction energy between regions I and II is computed by OFDFT. Although different levels of QM simulations could be employed in region I, we focus on KS-DFT and OFDFT in the present work. Similarly, while many empirical potentials could be used in region II, we choose embedded-atom method (EAM $)^{24}$ as an example of MM calculations in the paper. The total energy of the QM/MM system can be expressed as

$$
\begin{aligned}
E_{\mathrm{tot}}\left[\rho^{\mathrm{tot}} ; \mathbf{R}^{\mathrm{tot}}\right]= & \min _{\rho \mathrm{I}}\left\{E_{\mathrm{QM}}\left[\rho^{\mathrm{I}} ; \mathbf{R}^{\mathrm{I}}\right]+E_{\mathrm{OF}}^{\mathrm{int}}\left[\rho^{\mathrm{I}}, \rho^{\mathrm{II}} ; \mathbf{R}^{\mathrm{I}}, \mathbf{R}^{\mathrm{II}}\right]\right\} \\
& +E_{\mathrm{MM}}\left[\mathbf{R}^{\mathrm{II}}\right],
\end{aligned}
$$

where $\mathbf{R}^{\text {tot }} \equiv \mathbf{R}^{\mathrm{II}} \cup \mathbf{R}^{\mathrm{II}}, \mathbf{R}^{\mathrm{I}}$ and $\mathbf{R}^{\mathrm{II}}$ denote atomic coordinates in regions I and II, respectively. The total charge density of the system $\rho^{\text {tot }}$ consists of two contributions: $\rho^{\text {tot }}=\rho^{\mathrm{I}}+\rho^{\mathrm{II}}$, where $\rho^{\mathrm{I}}$ and $\rho^{\mathrm{II}}$ represent the charge density from regions I and II, respectively. While $\rho^{\mathrm{I}}$ is determined self-consistently by minimizing the total energy functional [Eq. (1)], $\rho^{\mathrm{II}}$ is defined as the superposition of atomic-centered charge densities $\rho^{\text {at }}$ via $\rho^{\mathrm{II}}(\mathbf{r})=\sum_{i \in \mathrm{II}} \rho^{\text {at }}\left(\mathbf{r}-\mathbf{R}_{i}\right)$. Noted that $\rho^{\text {at }}$ is spherically symmetric and can be constructed a priori. Therefore, $\rho^{\mathrm{II}}(\mathbf{r})$ is fixed for a given ionic configuration of region II, and it changes only upon the motion of region II ions. In other words, the electronic degree of freedom in the formulation is $\rho^{\mathrm{I}}$ only, and $\rho^{\mathrm{II}}$ is fixed during the electronic relaxation. The interaction energy formulated by OFDFT is written as follows:

$$
\begin{aligned}
E_{\mathrm{OF}}^{\mathrm{int}}\left[\rho^{\mathrm{I}}, \rho^{\mathrm{II}} ; \mathbf{R}^{\mathrm{I}}, \mathbf{R}^{\mathrm{II}}\right]= & E_{\mathrm{OF}}\left[\rho^{\mathrm{tot}} ; \mathbf{R}^{\mathrm{tot}}\right]-E_{\mathrm{OF}}\left[\rho^{\mathrm{I}} ; \mathbf{R}^{\mathrm{I}}\right] \\
& -E_{\mathrm{OF}}\left[\rho^{\mathrm{II}} ; \mathbf{R}^{\mathrm{II}}\right] .
\end{aligned}
$$

The corresponding embedding potential $\mu_{\mathrm{emb}}(\mathbf{r})$ is defined as

$$
\mu_{\mathrm{emb}}(\mathbf{r}) \equiv \frac{\delta E_{\mathrm{OF}}^{\mathrm{int}}\left[\rho^{\mathrm{I}}, \rho^{\mathrm{II}} ; \mathbf{R}^{\mathrm{I}}, \mathbf{R}^{\mathrm{II}}\right]}{\delta \rho^{\mathrm{I}}},
$$

which represents an effective single-particle potential that region I electrons feel due to the presence of region II.

A basic ansatz of the present formulation is that $\rho^{\mathrm{I}}$ must be confined within a finite volume $\left(\Omega^{\mathrm{I}}\right)$ that is necessarily larger than region I but much smaller than the entire system.

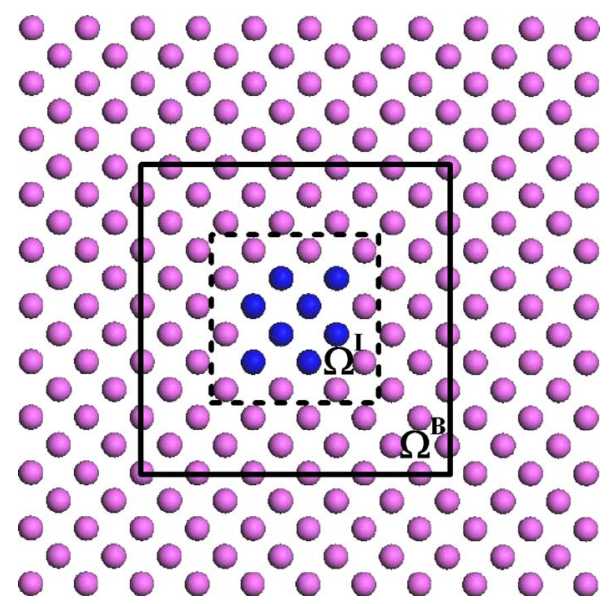

FIG. 1. (Color online) The schematic diagram of the partitioned system. Blue and magenta spheres represent the atoms belonging to region I and region II, respectively. The dashed box represents $\Omega^{\mathrm{I}}$, and the solid box represents the periodic box $\Omega^{\mathrm{B}}$ used for the evaluation of the kernel terms of the interaction energy.

As shown schematically in Fig. $1, \Omega^{\mathrm{I}}$ should include region I and extend several angstroms into region II. As a key computational parameter in the present formulation, $\Omega^{\mathrm{I}}$ needs to be carefully determined before simulations. Physically speaking, the overlap between $\rho^{\mathrm{I}}$ and $\rho^{\mathrm{II}}$ is a manifestation of the quantum coupling between the two regions.

According to OFDFT, the energy of an electronic system can be expressed as an explicit functional of electron density,

$$
E_{\mathrm{OF}}[\rho]=T_{\mathrm{s}}[\rho]+E_{\mathrm{H}}[\rho]+E_{\mathrm{e}-\mathrm{i}}[\rho]+E_{\mathrm{xc}}[\rho]+E_{\mathrm{i}-\mathrm{i}} .
$$

The various terms in Eq. (4) represent noninteracting kinetic energy, Hartree energy, electron-ion Coulomb energy, exchange-correlation energy, and ion-ion Coulomb energy, respectively. Here, $T_{\mathrm{s}}$ which is an explicit functional of electron density represents an approximation to the exact noninteracting kinetic energy. The state of the art of kinetic energy functionals is used in the present formulation which includes three contributions $T_{\mathrm{s}}[\rho]=T_{\mathrm{TF}}[\rho]+T_{\mathrm{vW}}[\rho]+T_{\mathrm{ker}}[\rho]$ (Refs. 22 and 25): Thomas-Fermi contribution $T_{\mathrm{TF}}$, exact for a uniform electron gas, ${ }^{26,27}$ von Weizsacher contribution $T_{\mathrm{vW}}$, exact for a single electron orbital, ${ }^{28}$ and a density-dependent convolution term $T_{\text {ker }}$ which ensures the correct linear response of the electron gas. ${ }^{22,29} E_{\mathrm{e}-\mathrm{i}}[\rho]$ is given by $\Sigma_{i} \int \rho(\mathbf{r}) V_{\mathrm{psp}}(\mathbf{r}$ $\left.-\mathbf{R}_{\mathrm{I}}\right) d \mathbf{r}$, and $V_{\text {psp }}$ is a local pseudopotential with a spherical symmetry. Local density approximation ${ }^{30}$ is used for $E_{\mathrm{xc}}[\rho]$. Finally, $E_{\mathrm{i}-\mathrm{i}}$ is given as $\sum_{i<j} \frac{Z_{i} Z_{j}}{\left|\mathbf{R}_{i}-\mathbf{R}_{j}\right|}$.

With Eqs. (2)-(4), one can express the interaction energy and the embedding potential as follows:

$$
\begin{gathered}
E_{\mathrm{OF}}^{\mathrm{int}}=T_{\mathrm{TF}}^{\mathrm{int}}+T_{\mathrm{vW}}^{\mathrm{int}}+T_{\mathrm{ker}}^{\mathrm{int}}+\left(E_{\mathrm{H}}^{\mathrm{int}}+E_{\mathrm{e}-\mathrm{i}}^{\mathrm{int}}+E_{\mathrm{i}-\mathrm{i}}^{\mathrm{int}}\right)+E_{\mathrm{xc}}^{\mathrm{int}}, \\
\mu_{\mathrm{emb}}=\mu_{\mathrm{TF}}^{\mathrm{emb}}+\mu_{\mathrm{vW}}^{\mathrm{emb}}+\mu_{\mathrm{ker}}^{\mathrm{emb}}+\mu_{\mathrm{H}, \mathrm{e}-\mathrm{i}, \mathrm{i}-\mathrm{i}}^{\mathrm{emb}}+\mu_{\mathrm{xc}}^{\mathrm{emb}},
\end{gathered}
$$

with various $\mu$ terms being the functional derivative of the corresponding interaction energy with respect to $\rho^{\mathrm{I}}$. As first pointed out by Choly et al. ${ }^{11}$ in addition to its computational efficiency, the OFDFT formulation of the interaction energy 
allows $E_{\mathrm{OF}}^{\text {int }}$ to be evaluated over $\Omega^{\mathrm{I}}$ rather than over the entire system as Eq. (2) appears to suggest. This represents a significant computational saving, thanks to the cancellation in evaluating the first and the third terms of Eq. (2) which is rendered by the orbital-free nature of OFDFT and the localization of $\rho^{\mathrm{I}}$ (i.e., $\rho^{\mathrm{I}}$ vanishes beyond $\Omega^{\mathrm{I}}$ ).

To see the cancellation explicitly, let us first consider the local terms of $E_{\mathrm{OF}}^{\text {int }}: T_{\mathrm{TF}}^{\mathrm{int}}, T_{\mathrm{vW}}^{\mathrm{int}}$, and $E_{\mathrm{xc}}^{\mathrm{int}}$. We take $T_{\mathrm{TF}}^{\mathrm{int}}$ as an example to demonstrate the cancellation and the other local terms work the same way.

$$
\begin{aligned}
T_{\mathrm{TF}}^{\mathrm{int}} & =\int f_{\mathrm{TF}}\left(\rho^{\mathrm{tot}}\right) d \mathbf{r}-\int f_{\mathrm{TF}}\left(\rho^{\mathrm{II}}\right) d \mathbf{r}-\int_{\Omega^{\mathrm{I}}} f_{\mathrm{TF}}\left(\rho^{\mathrm{I}}\right) d \mathbf{r} \\
& =\int_{\Omega^{\mathrm{I}}}\left[f_{\mathrm{TF}}\left(\rho^{\mathrm{tot}}\right)-f_{\mathrm{TF}}\left(\rho^{\mathrm{II}}\right)-f_{\mathrm{TF}}\left(\rho^{\mathrm{I}}\right)\right] d \mathbf{r},
\end{aligned}
$$

where $f_{\mathrm{TF}}(\rho)=\frac{3}{10}\left(3 \pi^{2}\right)^{2 / 3} \rho^{5 / 3}$, and we have used the fact that $\rho^{\mathrm{tot}}(\mathbf{r})=\rho^{\mathrm{II}}(\mathbf{r})$, when $\mathbf{r}$ is beyond $\Omega^{\mathrm{I}}$. Therefore, the calculation of $T_{\mathrm{TF}}^{\text {int }}$ involves an integral over $\Omega^{\mathrm{I}}$ rather than over the entire system.

Next, we examine the nonlocal terms of $E_{\mathrm{OF}}^{\text {int }}$ electrostatic terms $\left(E_{\mathrm{H}}^{\mathrm{int}}+E_{\mathrm{e}-\mathrm{i}}^{\mathrm{int}}+E_{\mathrm{i}-\mathrm{i}}^{\mathrm{int}}\right)$ and the kernel term $T_{\mathrm{ker}}^{\mathrm{int}}$ of the kinetic energy. For the electrostatic terms, the long-range $1 / R$ Coulomb interactions in electron-electron, electron-ion, and ionion contributions are cancelled identically for a chargeneutral system. Thus, it is convenient to group the three terms together when evaluating the electrostatic terms. The sum of the three terms can be expressed as

$$
\begin{aligned}
\left(E_{\mathrm{H}}^{\mathrm{int}}+E_{\mathrm{e}-\mathrm{i}}^{\mathrm{int}}+E_{\mathrm{i}-\mathrm{i}}^{\mathrm{int}}\right)= & \int_{\Omega^{\mathrm{I}}} \rho^{\mathrm{I}}(\mathbf{r})\left[\sum_{j} V_{\mathrm{elec}}^{\mathrm{at}}\left(\mathbf{r}-\mathbf{R}_{j}^{\mathrm{II}}\right)\right] d \mathbf{r} \\
& +\sum_{i, j} \chi\left(\mathbf{R}_{i}^{\mathrm{I}}-\mathbf{R}_{j}^{\mathrm{II}}\right), \\
\mu_{\mathrm{H}, \mathrm{e}-\mathrm{i}, \mathrm{i}-\mathrm{i}}^{\mathrm{ei}} & =\sum_{j} V_{\mathrm{elec}}^{\mathrm{at}}\left(\mathbf{r}-\mathbf{R}_{j}^{\mathrm{II}}\right),
\end{aligned}
$$

where we define

$$
\begin{gathered}
V_{\mathrm{elec}}^{\mathrm{at}}(\mathbf{r}) \equiv \int \frac{\rho^{\mathrm{at}}\left(\mathbf{r}^{\prime}\right)}{\left|\mathbf{r}-\mathbf{r}^{\prime}\right|} d \mathbf{r}^{\prime}+V_{\mathrm{psp}}(\mathbf{r}), \\
\chi\left(\mathbf{R}_{i}^{\mathrm{I}}-\mathbf{R}_{j}^{\mathrm{II}}\right) \equiv \int V_{\mathrm{psp}}\left(\mathbf{r}-\mathbf{R}_{i}^{\mathrm{I}}\right) \rho^{\mathrm{at}}\left(\mathbf{r}-\mathbf{R}_{j}^{\mathrm{II}}\right) d \mathbf{r}+\frac{Z_{i} Z_{j}}{\left|\mathbf{R}_{i}^{\mathrm{I}}-\mathbf{R}_{j}^{\mathrm{II}}\right|} .
\end{gathered}
$$

Here, $V_{\text {elec }}^{\text {at }}\left(\mathbf{r}-\mathbf{R}_{j}^{\mathrm{II}}\right)$ represents the Coulomb potential at $\mathbf{r}$ in $\Omega^{\mathrm{I}}$ due to the $j$ th atom in region II, and $\chi\left(\mathbf{R}_{i}^{\mathrm{I}}-\mathbf{R}_{j}^{\mathrm{II}}\right)$ represents the Coulomb interaction between the $i$ th ion in region I and the $j$ th atom in region II. Thanks to the spherical symmetry of $\rho^{\text {at }}$ and $V_{\mathrm{psp}} ; V_{\text {elec }}^{\text {at }}$ and $\chi$ are functions of distance. Due to $V_{\text {elec }}^{\text {at }}(\mathbf{r})$ which is the electrostatic potential of a neutral atom and $\chi(\mathbf{R})$ which is the interaction between an ion and a neutral atom, both of them are short-ranged functions. Therefore, similar to the local terms, the calculation of the electrostatic terms is also confined to a smaller volume than the entire system. The extend of the volume is determined by a cutoff distance beyond which the $1 / R$ relation is exact. The actual calculation of the electrostatic terms is performed in real space.

However, the kernel term of the kinetic energy has a complicated nonlocal form which requires a close scrutiny,

$$
\begin{aligned}
T_{\mathrm{ker}}^{\mathrm{int}}=\int_{\Omega^{\mathrm{I}}} F(\mathbf{r})(K * G)(\mathbf{r}) d \mathbf{r}+\int_{\Omega^{\mathrm{I}}} F(\mathbf{r})\left(K * g_{2}\right)(\mathbf{r}) d \mathbf{r} \\
+\int_{\Omega^{\mathrm{I}}} G(\mathbf{r})\left(K * f_{2}\right)(\mathbf{r}) d \mathbf{r}-\int_{\Omega^{\mathrm{I}}} f_{1}(\mathbf{r})\left(K * g_{1}\right)(\mathbf{r}) d \mathbf{r}, \\
\mu_{\mathrm{ker}}^{\mathrm{emb}}=F^{\prime}(\mathbf{r})(K * G)(\mathbf{r})+G^{\prime}(\mathbf{r})(K * F)(\mathbf{r}) \\
+F^{\prime}(\mathbf{r})\left(K * g_{2}\right)(\mathbf{r})+G^{\prime}(\mathbf{r})\left(K * f_{2}\right)(\mathbf{r}) \\
-f_{1}^{\prime}(\mathbf{r})\left(K * g_{1}\right)(\mathbf{r})-g_{1}^{\prime}(\mathbf{r})\left(K * f_{1}\right)(\mathbf{r}),
\end{aligned}
$$

where the convolution integral is defined as $\left(K^{*} G\right)(\mathbf{r})$ $\equiv \int K\left(\mathbf{r}-\mathbf{r}^{\prime}\right) G\left(\mathbf{r}^{\prime}\right) d \mathbf{r}^{\prime}$, etc., and

$$
\begin{gathered}
f_{1}(\mathbf{r}) \equiv f\left[\rho^{\mathrm{I}}(\mathbf{r})\right], \quad f_{12}(\mathbf{r}) \equiv f\left[\rho^{\mathrm{I}}(\mathbf{r})+\rho^{\mathrm{II}}(\mathbf{r})\right], \\
g_{1}(\mathbf{r}) \equiv g\left[\rho^{\mathrm{I}}(\mathbf{r})\right], \quad g_{12}(\mathbf{r}) \equiv g\left[\rho^{\mathrm{I}}(\mathbf{r})+\rho^{\mathrm{II}}(\mathbf{r})\right], \\
F(\mathbf{r}) \equiv f_{12}(\mathbf{r})-f_{2}(\mathbf{r}), \quad G(\mathbf{r}) \equiv g_{12}(\mathbf{r})-g_{2}(\mathbf{r}) .
\end{gathered}
$$

The details of the density-dependent kernel terms can be found elsewhere. ${ }^{22,25}$ As shown in Eq. (9), although $T_{\mathrm{ker}}^{\mathrm{int}}$ is expressed as integrals over $\Omega^{\mathrm{I}}$, the time-consuming convolution terms, such as $\left(K^{*} f_{2}\right)(\mathbf{r})$, are actually integrated over the entire space. The efficient treatment of these convolution terms will be discussed in Sec. III A.

The ionic force is calculated by varying the total energy with respect to the ionic displacement. At the ground state of the electrons, $\rho_{\text {min }}^{\mathrm{I}}$ satisfies $\delta E_{\text {tot }} /\left.\delta \rho^{\mathrm{I}}\right|_{\rho_{\min }^{\mathrm{I}}}=0$; therefore, the ionic force in region I $\left(\mathbf{F}_{i \in \mathrm{I}}\right)$ and region II $\left(\mathbf{F}_{j \in \mathrm{II}}\right)$ can be written as

$$
\begin{gathered}
-\mathbf{F}_{i \in \mathrm{I}}=\frac{\partial E_{\mathrm{QM}}}{\partial \mathbf{R}_{i}}+\frac{\partial E_{\mathrm{OF}}^{\mathrm{int}}}{\partial \mathbf{R}_{i}}, \\
-\mathbf{F}_{j \in \mathrm{II}}=\frac{\partial E_{\mathrm{OF}}^{\mathrm{int}}}{\partial \mathbf{R}_{j}}+\frac{\partial E_{\mathrm{MM}}}{\partial \mathbf{R}_{j}} .
\end{gathered}
$$

Here, $-\frac{\partial E_{\mathrm{QM}}}{\partial \mathbf{R}_{i}}$ and $-\frac{\partial E_{\mathrm{MM}}}{\partial \mathbf{R}_{j}}$ can be calculated by standard atomistic methods. The force on the $i$ th ion in region I due to the interaction energy is given by

$$
\begin{aligned}
-\mathbf{F}_{i \in \mathrm{I}}^{\mathrm{int}} & =\frac{\partial E_{\mathrm{OF}}^{\mathrm{int}}}{\partial \mathbf{R}_{i}} \\
& =\sum_{j \in \mathrm{II}} \nabla_{\mathbf{R}_{i}} \chi\left(\mathbf{R}_{i}-\mathbf{R}_{j}\right) \\
& =\sum_{j \in \mathrm{II}} \chi^{\prime}\left(\left|\mathbf{R}_{i}-\mathbf{R}_{j}\right|\right) \frac{\left(\mathbf{R}_{i}-\mathbf{R}_{j}\right)}{\left|\mathbf{R}_{i}-\mathbf{R}_{j}\right|} .
\end{aligned}
$$

Moreover, $\mathbf{F}_{i \in \mathrm{I}}^{\mathrm{int}}$ is short ranged, and only the region II ions which are adjacent to the QM/MM interface have appreciable contributions. The force expression on region II ions, on the other hand, is much more complicated because its 
energy is not minimized with respect to $\rho^{\mathrm{II}}$, and thus, there are force contributions from the variation of $\rho^{\mathrm{II}}$. The force on the $j$ th ion in region II due to the interaction energy is given by

$$
-\mathbf{F}_{j \in \mathrm{II}}^{\mathrm{int}}=\frac{\partial E_{\mathrm{TF}+\mathrm{vW}+\mathrm{ker}}^{\mathrm{int}}}{\partial \mathbf{R}_{j}}+\frac{\partial\left[E_{\mathrm{H}}^{\mathrm{int}}+E_{\mathrm{e}-\mathrm{i}}^{\mathrm{int}}+E_{\mathrm{i}-\mathrm{i}}^{\mathrm{int}}\right]}{\partial \mathbf{R}_{j}}+\frac{\partial E_{\mathrm{xc}}^{\mathrm{int}}}{\partial \mathbf{R}_{j}},
$$

where the various contributions of the first term are given as

$$
\begin{gathered}
\frac{\partial T_{\mathrm{TF}}^{\mathrm{int}}}{\partial \mathbf{R}_{j}}=\int_{\Omega^{\mathrm{I}}} \nabla_{\mathbf{R}_{j}} \rho^{\mathrm{at}}\left(\mathbf{r}-\mathbf{R}_{j}\right)\left[\mu_{\mathrm{TF}}\left(\rho^{\mathrm{I}}+\rho^{\mathrm{II}}\right)-\mu_{\mathrm{TF}}\left(\rho^{\mathrm{II}}\right)\right] d \mathbf{r}, \\
\frac{\partial T_{\mathrm{vW}}^{\mathrm{int}}}{\partial \mathbf{R}_{j}}=\int_{\Omega^{\mathrm{I}}} \nabla_{\mathbf{R}_{j}} \rho^{\mathrm{at}}\left(\mathbf{r}-\mathbf{R}_{j}\right)\left[\mu_{\mathrm{vW}}\left(\rho^{\mathrm{I}}+\rho^{\mathrm{II}}\right)-\mu_{\mathrm{vW}}\left(\rho^{\mathrm{II}}\right)\right] d \mathbf{r}, \\
\frac{\partial T_{\mathrm{ker}}^{\mathrm{int}}}{\partial \mathbf{R}_{j}}=\int_{\Omega^{\mathrm{I}}} \nabla_{\mathbf{R}_{j}} \rho^{\mathrm{at}}\left(\mathbf{r}-\mathbf{R}_{j}\right)\left[f_{12}^{\prime}(\mathbf{r})(K * G)(\mathbf{r})+g_{12}^{\prime}(\mathbf{r})(K * F)\right. \\
\left.\quad \times(\mathbf{r})+F^{\prime}(\mathbf{r})\left(K * g_{2}\right)(\mathbf{r})+G^{\prime}(\mathbf{r})\left(K * f_{2}\right)(\mathbf{r})\right] d \mathbf{r} . \quad(14)
\end{gathered}
$$

The second term is given by

$$
\begin{aligned}
& \frac{\partial\left[E_{\mathrm{H}}^{\mathrm{int}}+E_{\mathrm{e}-\mathrm{i}}^{\mathrm{int}}+E_{\mathrm{i}-\mathrm{i}}^{\mathrm{int}}\right]}{\partial \mathbf{R}_{j}} \\
& \quad=\sum_{i \in \mathrm{I}} \nabla_{\mathbf{R}_{j}} \chi\left(\mathbf{R}_{i}-\mathbf{R}_{j}\right)+\int_{\Omega^{\mathrm{I}}} \rho^{\mathrm{I}}(\mathbf{r}) \nabla_{\mathbf{R}_{j}} V_{\text {elec }}^{\text {at }}\left(\mathbf{r}-\mathbf{R}_{j}\right) d \mathbf{r},
\end{aligned}
$$

and the third term is given by

$$
\frac{\partial\left[E_{\mathrm{xc}}^{\mathrm{int}}\right]}{\partial \mathbf{R}_{j}}=\int_{\Omega^{\mathrm{I}}} \nabla_{\mathbf{R}_{j}} \rho^{\mathrm{at} t}\left(\mathbf{r}-\mathbf{R}_{j}\right)\left[\mu_{\mathrm{xc}}\left(\rho^{\mathrm{I}}+\rho^{\mathrm{II}}\right)-\mu_{\mathrm{xc}}\left(\rho^{\mathrm{II}}\right)\right] d \mathbf{r} .
$$

All the integrations are performed within $\Omega^{\mathrm{I}}$, and $\mathbf{F}_{j \in \text { II }}^{\mathrm{int}}$ is nonzero only for the region II ions which are adjacent to the interface.

Since region I requires a much longer computation than region II, the following algorithm allows for an efficient ionic relaxation: ${ }^{11}$ we denote $\mathbf{R}^{\mathrm{I}^{\prime}}$ the set of region I ions plus all region II ions $\mathbf{R}^{\mathrm{II}}$ for which the interaction force $\partial E_{\mathrm{OF}}^{\mathrm{int}} / \partial \mathbf{R}_{j}^{\mathrm{II}}$ is not negligible, and we denote $\mathbf{R}^{\mathrm{II}}$ the rest of region II ions. The relaxation algorithm is as follows:

(i) Minimize $E_{\text {tot }}$ with respect to $\mathbf{R}^{\mathrm{II}^{\prime}}$ while holding $\mathbf{R}^{\mathrm{I}^{\prime}}$ fixed. This only involves the MM calculation.

(ii) Perform one step of conjugate-gradient minimization of $E_{\text {tot }}$ with respect to $\mathbf{R}^{\mathbf{I}^{\prime}}$ with $\mathbf{R}^{\mathrm{II}}$ fixed.

(iii) Repeat until the entire system is relaxed.

In this manner, the number of QM calculations performed is greatly reduced, albeit at the expense of more MM calculations.

\section{IMPLEMENTATIONS}

In this section, we will discuss the implementation of the present QM/MM method, focusing on several crucial com- ponents of the method. In specific, we have implemented two QM methods for the calculations of $E_{\mathrm{QM}}\left[\rho^{\mathrm{I}} ; \mathbf{R}^{\mathrm{I}}\right]$ : OFDFT and KS-DFT. While the degree of freedom in the first method (OFDFT) is electron density $\rho^{\mathrm{I}}$, the degrees of freedom in the second method (KS-DFT) are KS orbitals. Since the computational cost of OFDFT is much smaller than that of KSDFT, the first method can deal with a much larger QM region. However, the application of the first method is limited by the applicability of the kinetic energy functionals and local pseudopotentials. Reliable kinetic energy functionals and local pseudopotentials exist only for a handful of elements. The second method, although cannot treat a very large QM region, it could be used for a wide variety of materials systems.

In this paper, we use aluminum as a test material for which excellent kinetic energy functional and local pseudopotential do exist. In the first method, the parameters of the density-dependent kernel are chosen from Ref. 22, and Al ions are represented by Goodwin-Needs-Heine local pseudopotential. ${ }^{31}$ In the second method, we use the Vienna ab initio simulation package (VASP) $)^{32-34}$ which is based on KS-DFT for QM calculations. The "force-matching" empirical EAM, potential of $\mathrm{Al}^{35,36}$ is used for MM simulations. The potential has been scaled to yield precisely the same lattice constant and bulk module as those determined by the corresponding KS-DFT and OFDFT calculations. This procedure is important for reducing coupling errors.

\section{A. Orbital-free density functional theory for region I}

In this section, we first examine the plausibility of employing periodic boundary conditions for computing the kernel terms in $T_{\mathrm{ker}}^{\mathrm{int}}$. We then discuss how atomiclike charge density $\rho^{\text {at }}(r)$ can be constructed to ensure satisfactory coupling. Finally, we propose a method to eliminate the socalled "ghost" force on region II ions.

\section{Fourier transformation for the kernel terms}

As seen in Eq. (9), the most time-consuming part of the calculation is the convolution integrals. If fast Fourier transformations (FFTs) could be invoked to evaluate the convolution integrals, the computational saving could be significant: $\hat{\mathcal{F}}\left[\left(K * f_{2}\right)(\mathbf{r})\right]=\hat{\mathcal{F}}[K(\mathbf{r})] \hat{\mathcal{F}}\left[f_{2}(\mathbf{r})\right]$. However, application of FFT requires that periodic boundary conditions be employed over a finite domain or a periodic box. This leads to potential problems because the convolutions in question should be performed over the whole space. Therefore, it appears that FFT operations on the kernel terms could lead to errors. In the following, we will examine whether the errors are small enough to warrant the FFT calculations of the convolutions. We will use $\left(K * f_{2}\right)(\mathbf{r})$ as an example. The integration over the whole space can be divided into two parts:

$$
\begin{aligned}
\left(K * f_{2}\right)(\mathbf{r}) \equiv & \int K\left(\mathbf{r}-\mathbf{r}^{\prime}\right) f_{2}\left(\mathbf{r}^{\prime}\right) d \mathbf{r}^{\prime} \\
= & \int_{\Omega^{\mathrm{B}}} K\left(\mathbf{r}-\mathbf{r}^{\prime}\right) f_{2}\left(\mathbf{r}^{\prime}\right) d \mathbf{r}^{\prime} \\
& +\int_{\Omega^{\mathrm{B}^{\prime}}} K\left(\mathbf{r}-\mathbf{r}^{\prime}\right) f_{2}\left(\mathbf{r}^{\prime}\right) d \mathbf{r}^{\prime},
\end{aligned}
$$




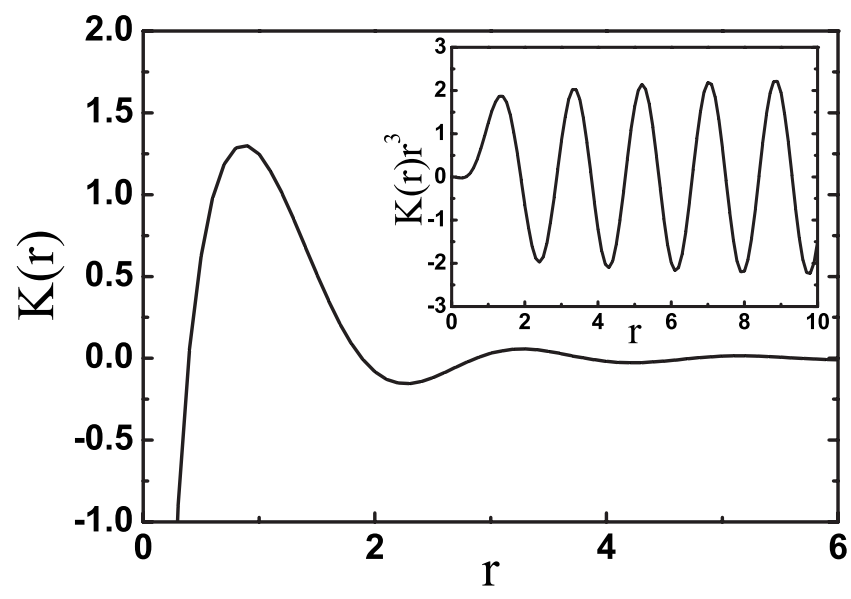

FIG. 2. Kernel function $K(r)$ versus $r(\AA)$. Inset: the plot of $K(r) r^{3}$ versus $r$.

where $\Omega^{\mathrm{B}}$ represents the periodic box over which FFT is performed. $\Omega^{\mathrm{B}^{\prime}}$ denotes the space beyond the periodic box. Let us compare two ways of calculating the convolution: exact versus FFT. In the exact calculation, the space of integration should be $\Omega^{\mathrm{B}} \cup \Omega^{\mathrm{B}^{\prime}}$, while with FFT, the space becomes $\Omega^{\mathrm{B}}$ along with its periodic images. Therefore, although the first term of Eq. (17) is calculated correctly by FFT, the calculation of the second term is in error. Since $\mathbf{r}$ $\in \Omega^{\mathrm{I}}$ [see Eq. (9)] and $\mathbf{r}^{\prime} \in \Omega^{\mathrm{B}^{\prime}}$ in the second term, the error associated with FFT is dictated by the decaying property of $K(r)$. If $K(r)$ decays fast enough, the error in the second term could become negligible.

Let us now consider the decaying property of $K(r)$, which is a nontrivial Fourier transform of $K(q)$. By using the numerical results of Herring, ${ }^{37}$ we plot $K(r)$ versus $r$ in Fig. 2, which suggests that $K(r)$ decays as $r^{-3}$. In principle, one should keep $\left|\mathbf{r}-\mathbf{r}^{\prime}\right|$ large enough so that the error in the second term becomes negligible. However, this demands a very large periodic box $\Omega^{\mathrm{B}}$ which could render the calculation intractable. We thus need to optimize the size of the periodic box so that the computation is both accurate and efficient.
For this purpose, we perform the following test in a perfect Al lattice. If the $\mathrm{QM} / \mathrm{MM}$ coupling were perfect, the force and displacement on each ion in the test system would be identically zero since regions I and II have the same lattice constant. In other words, any nonzero force or displacement is indicative of coupling errors. One can thus estimate the coupling errors by examining the magnitude of force or displacement on each ions in the test system. We denote $F_{\text {max }}^{\mathrm{I}}$ $\left(F_{\max }^{\mathrm{II}}\right)$ as the maximum force on region I (II) atoms, and $d_{\max }^{\max }$ $\left(d_{\max }^{\mathrm{II}}\right)$ as the maximum displacement on region I (II) atoms, respectively. The relaxation for all ions is performed until the maximum force on any ion is less than $0.01 \mathrm{eV} / \AA$. In the test, we select two different periodic boxes with sizes of $6 a_{0} \times 6 a_{0} \times a_{0}$ and $5.5 a_{0} \times 5.5 a_{0} \times a_{0}$, respectively $\left(a_{0}\right.$ is the lattice constant). The first case corresponds to a "correct" choice of $\Omega^{\mathrm{B}}$ since it is an integer multiple of the lattice constant. The total charge density constructed with the periodic boundary conditions matches well the real total charge density of the test system. On the other hand, the second case represents an "incorrect" (in fact, the "worst") choice of $\Omega^{\mathrm{B}}$ because it is an half-integer multiple of the lattice constant; thus, the error of $\rho^{\mathrm{II}}\left(\mathbf{r} \in \Omega^{\mathrm{B}^{\prime}}\right)$ reaches maximum for the test system. In this way, we intentionally introduce large errors of charge density around the edge of $\Omega^{\mathrm{B}}$, and we want to examine how the errors due to the periodic boundary conditions are translated to the coupling errors.

The test results of $F_{\max }^{\mathrm{I}}, F_{\max }^{\mathrm{II}}, d_{\max }^{\mathrm{I}}$, and $d_{\max }^{\mathrm{II}}$ are summarized in Table I. From the comparisons of system No. 3 and No. 4, we find that the two cases produce the identical coupling errors, which are very small. The test results strongly suggest that (1) the size of $\Omega^{\mathrm{B}}$ is large enough to yield small coupling errors for the test system; (2) for a sufficiently large $\Omega^{\mathrm{B}}$, the errors in convolution terms due to FFT are essentially zero, thanks to the fast decaying property of $K(r)$. Therefore, we establish that FFT could be used to evaluate $T_{\mathrm{ker}}^{\mathrm{int}}$ for a modest value of $\Omega^{\mathrm{B}}$. The present FFT approach represents a significant improvement over the original method of Choly et al. in two aspects: (1) it allows for a much more efficient calculation of the time-consuming term $T_{\mathrm{ker}}^{\mathrm{int}}$; (2) it allows for more accurate computation of $T_{\mathrm{ker}}^{\mathrm{int}}$ than

TABLE I. Test results of $F_{\max }^{\mathrm{I}}$ and $F_{\max }^{\mathrm{II}}(\mathrm{ev} / \AA)$ and $d_{\max }^{\mathrm{I}}$ and $d_{\max }^{\mathrm{II}}(\AA)$. The details of calculations are summarized in the column of remark as $\mathbf{a}$, the first coupling method; $\mathbf{b}$, the second coupling method; $\mathbf{c}$, entire system consisting of $14 a_{0} \times 14 a_{0} \times a_{0}$ with the innermost $2 a_{0} \times 2 a_{0} \times a_{0}$ as region I; d, entire system consisting of $14 a_{0} \times 14 a_{0} \times 14 a_{0}$ with the innermost $2 a_{0} \times 2 a_{0} \times 2 a_{0}$ as region I; e, the periodic box size chosen as $6 a_{0} \times 6 a_{0} \times a_{0}$; $\mathbf{f}$, the periodic box size chosen as $6 a_{0} \times 6 a_{0} \times 6 a_{0}$; $\mathbf{g}$, the periodic box size chosen as $5.5 a_{0} \times 5.5 a_{0} \times a_{0} ; \mathbf{h}$, atomic charge density is constructed by SSTO; $\mathbf{i}$, atomic charge density is constructed by MSTO; $\mathbf{j}$, with optimized volume of $\Omega^{\mathrm{I}} ; \mathbf{k}$, with the correction force; and $\mathbf{l}$, the best results from Ref. 11.

\begin{tabular}{lccccc}
\hline \hline System & Remark & $F_{\max }^{\mathrm{I}}$ & $F_{\max }^{\mathrm{II}}$ & $d_{\max }^{\mathrm{I}}$ & $d_{\max }^{\mathrm{II}}$ \\
\hline 1 & $\mathbf{a}, \mathbf{c}, \mathbf{e}, \mathbf{h}, \mathbf{j}$ & 0.013 & 0.132 & 0.044 & 0.116 \\
2 & $\mathbf{a}, \mathbf{c}, \mathbf{e}, \mathbf{i}, \mathbf{j}$ & 0.03 & 0.132 & unconverged & unconverged \\
3 & $\mathbf{a}, \mathbf{c}, \mathbf{e}, \mathbf{h}, \mathbf{j}, \mathbf{k}$ & 0.013 & 0.000 & 0.002 & 0.000 \\
4 & $\mathbf{a}, \mathbf{c}, \mathbf{g}, \mathbf{h}, \mathbf{j}, \mathbf{k}$ & 0.013 & 0.000 & 0.002 & 0.000 \\
5 & $\mathbf{a}, \mathbf{d}, \mathbf{f}, \mathbf{h}, \mathbf{j}, \mathbf{k}$ & 0.024 & 0.000 & 0.009 & 0.002 \\
6 & $\mathbf{b}, \mathbf{c}, \mathbf{e}, \mathbf{h}, \mathbf{j}, \mathbf{k}$ & 0.065 & 0.000 & 0.009 & 0.004 \\
7 & $\mathbf{a}, \mathbf{d}, \mathbf{l}$ & 0.09 & 0.37 & 0.22 & 0.26 \\
\hline \hline
\end{tabular}


the original method which was formulated approximately in real space.

\section{Atomic charge density}

Since $\rho^{\mathrm{II}}$ provides the boundary conditions for which the OFDFT equation of $\rho^{\mathrm{I}}$ is solved, it is crucial to construct an appropriate representation of $\rho^{\mathrm{II}}$. In fact, constructing an appropriate charge density distribution in region II is a common challenge to many QM/MM methods. ${ }^{2}$ In this paper, we represent $\rho^{\mathrm{II}}$ as a superposition of spherical atomiclike charge densities centered on each ions in region II, which is a good approximation for metallic systems. Ideally, the superposition of $\rho^{\text {at }}(r)$ should reproduce the bulk charge density obtained by a QM calculation of the perfect lattice. That is to say, one needs to determine $\rho^{\text {at }}(r)$ by minimizing the following function:

$$
\int_{\mathrm{V}_{\mathrm{u}}}\left[\sum_{\mu} \rho^{\mathrm{at}}\left(\mathbf{r}-\mathbf{R}_{\mu}\right)-\rho^{\mathrm{latt}}(\mathbf{r})\right]^{2} d \mathbf{r},
$$

here, $V_{\mathrm{u}}$ represents the volume of the unit cell and $\rho^{\text {latt }}$ is the charge density obtained by an OFDFT calculation for the bulk reference system. $\mathbf{R}_{\mu}$ denotes the coordinate of the $\mu$ th ion.

By Fourier transform of Eq. (18), Choly et al. obtained the atomic charge density in reciprocal space as $\rho^{\text {at }}(q)$ $=\left\langle\rho_{\mathbf{q}}^{\text {latt }}\right\rangle_{q} /\left\langle S_{\mathbf{q}}\right\rangle_{q}$. Here, $S_{\mathbf{q}}$ is the structure factor, and $\langle\ldots\rangle_{q}$ represents an average over all reciprocal lattice vectors of the same length $q . \rho^{\text {at }}(q)$ was then used to construct the radial charge density $\rho^{\text {at }}(r)$. However, for a fcc structure, $S_{\mathbf{q}}$ vanishes for many $\mathbf{q}$. Therefore, only a handful of $\mathbf{q}$ could be included in the calculation, and thus the numerical error was considerable. In this work, we employ a parametrized Slatertype orbital to expand $\rho^{\text {at }}(r)$. One could use either single Slater-type orbital (SSTO) or multiple Slater-type orbitals (MSTOs) for the expansion. The SSTO is given by

$$
\rho^{\mathrm{at}}\left(r, n_{\mathrm{p}}\right)=A r^{2\left(n_{\mathrm{p}}-1\right)} e^{-\xi r}
$$

where $n_{\mathrm{p}}$ is the principle quantum number and $A$ and $\xi$ are parameters to be determined. The $\mathrm{MSTO}^{38}$ is given by $\rho^{\text {at }}(r)=\rho_{s}(r)+\rho_{p}(r)$, and for $\mathrm{Al}$, the spherically averaged $s$-like density is given by

$$
\rho_{s}(r)=\left|\sum_{i} A_{i} \frac{\left(2 \xi_{i}\right)^{n_{i+1 / 2}}}{\sqrt{\left(2 n_{i}\right) !}} r^{n_{i}-1} e^{-\xi_{i} r}\right|^{2} / 4 \pi,
$$

where $A_{i}, \xi_{i}$, and $n_{i}$ are also unknown parameters to be determined. Similar equation for $\rho_{p}(r)$ is skipped here in the interest of space. All the unknown parameters are determined by solving Eq. (20) with the constraint of preserving the correct number of valence electrons. The results of $\rho^{\text {at }}(r)$ for both choices are shown in Fig. 3. Due to the unphysical kinks in $\rho^{\text {at }}(r)$, MSTO gives a poor result in test (see Table I for system No. 2). By comparing the results for system No. 1 and No. 7, we conclude that SSTO is a better choice for expanding $\rho^{\text {at }}(r)$.

Next, we touch upon the selection of the all-important computational parameter $\Omega^{\mathrm{I}}$ : it should be large enough to accommodate $\rho^{\mathrm{I}}$ completely, but it cannot be too large; oth-

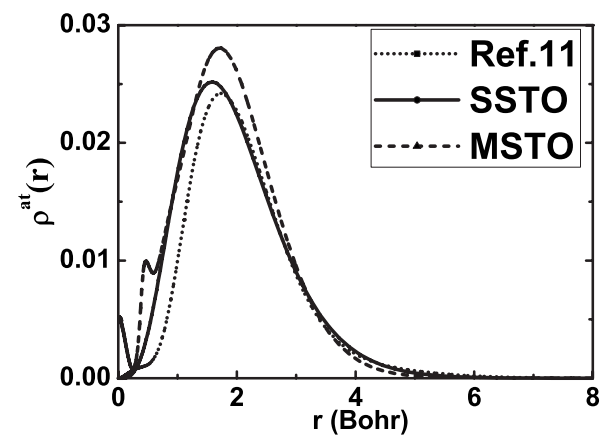

FIG. 3. Atomic charge density $\rho^{\text {at }}(r)$ as a function of radial distance by using SSTO (solid line) and MSTO (dashed line). The result from Ref. 11 is also included here (dotted line) for comparison.

erwise the correct $\rho^{\mathrm{I}}$ may not be obtainable with local minimization techniques. For $\mathrm{Al}$, we find an optimal value of $2.81 \AA$ for the distance between the boundaries of region I and $\Omega^{\mathrm{I}}$.

In Fig. 4, we present the total charge density with $\rho^{\text {at }}$ expanded by SSTO. The total charge density reproduces very well the correct charge density for the entire system, and there is a smooth transition across the QM/MM boundarythere is no visible interface between the two regions in terms of electron density.

Finally, we comment on the possibility of using Wannier functions to construct $\rho^{\mathrm{II}}$. Since under normal conditions, region II should be defect-free; Wannier functions ${ }^{39}$ or their maximally localized cousins ${ }^{40}$ should be a good choice for constructing $\rho^{\text {at }}(\mathbf{r})$. One can first obtain the Bloch wave functions $\phi_{n}(\mathbf{k}, \mathbf{r})$ ( $n$ is band index) from a plane-wave KS-DFT calculation for a unit cell. The Wannier functions are then given by

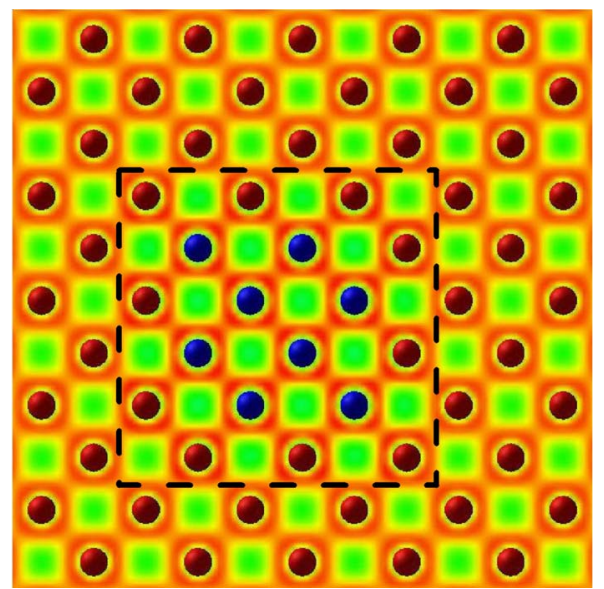

FIG. 4. (Color online) Total charge density $\rho^{\text {tot }}$ (in $\AA^{-3}$ ) for $\mathrm{Al}$ perfect lattice obtained by using the first coupling method. Contour scale ranges from 0.0027 (blue) to 0.0336 (red). Blue and red spheres represent ions belonging to regions I and II, respectively. The dashed box denotes the boundary of $\Omega^{\mathrm{I}}$. 


$$
W_{n}(\mathbf{R}, \mathbf{r})=\frac{V}{(2 \pi)^{3}} \int d \mathbf{k} \phi_{n}(\mathbf{k}, \mathbf{r}) e^{-i \mathbf{k} \cdot \mathbf{R}},
$$

where $\mathbf{R}$ is the ionic position and $V$ is the volume of the unit cell. Finally, the atomiclike charge density can be calculated as

$$
\rho_{\mathrm{w}}^{\mathrm{at}}(\mathbf{r}-\mathbf{R})=\sum_{n}\left|W_{n}(\mathbf{R}, \mathbf{r})\right|^{2}
$$

Notice that $\rho_{\mathrm{w}}^{\mathrm{at}}$ is no longer spherically symmetric. The desirable $\rho^{\mathrm{II}}$ can thus be constructed by the superposition of $\rho_{\mathrm{w}}^{\mathrm{at}}$, and $\rho^{\mathrm{II}}$ would be exact for a perfect lattice.

\section{Ghost force correction}

From Table I, we find that for system No. $1, F_{\max }^{\mathrm{II}}$ is much larger than $F_{\max }^{\mathrm{I}}$. The reason for the marked difference is as follows: the energetic formulation for region I is OFDFT, which is the same for the interaction energy; for region II, however, the energetic formulation is EAM, different from that of the interaction energy. This disparity in energetic formulation of region II leads to a disparity in force formulation, which causes additional coupling errors. The force errors due to disparate energetic formulations in a multiscale system are sometimes referred as "ghost force" in literature 4 - the terminology we will use in the paper.

To eliminate the ghost force on region II ions, we remove the energetic disparity by replacing the OFDFT calculation of the interaction energy by EAM. Note that this change of formulation is only applied to force calculation on region II ions; the total energy of system and the force calculation on region I ions remain the same. With this modification, the force on region II ions becomes $\mathbf{F}_{j \in \text { II }}^{*}$ which defined as

$$
-\mathbf{F}_{j \in \mathrm{II}}^{*}=\frac{\partial E_{\mathrm{EAM}}^{\mathrm{int}}}{\partial \mathbf{R}_{j}}+\frac{\partial E_{\mathrm{EAM}}}{\partial \mathbf{R}_{j}} .
$$

Associated with this modification, we introduce a correction force $\Delta \mathbf{F}_{j \in \text { II }}^{\text {corr }}$ as

$$
\Delta \mathbf{F}_{j \in \mathrm{II}}^{\mathrm{corr}}=\mathbf{F}_{j}^{*}-\mathbf{F}_{j}=\frac{\partial\left(E_{\mathrm{OF}}^{\mathrm{int}}-E_{\mathrm{EAM}}^{\mathrm{int}}\right)}{\partial \mathbf{R}_{j}} .
$$

Finally, we modify the total energy expression so that it is consistent to the force correction introduced above,

$$
\begin{aligned}
& E_{\text {tot }}^{*}\left[\mathbf{R}^{\text {tot }}\right]=\min _{\rho^{\mathrm{I}}}\left\{E_{\mathrm{OF}}\left[\rho^{\mathrm{I}} ; \mathbf{R}^{\mathrm{I}}\right]+E_{\mathrm{OF}}^{\mathrm{int}}\left[\rho^{\mathrm{I}}, \rho^{\mathrm{II}} ; \mathbf{R}^{\mathrm{I}}, \mathbf{R}^{\mathrm{II}}\right]\right\} \\
& +E_{\mathrm{EAM}}\left[\mathbf{R}^{\mathrm{II}}\right]-\sum_{j \in \mathrm{II}} \Delta \mathbf{F}_{j}^{\mathrm{corr}} \cdot \Delta \mathbf{R}_{j},
\end{aligned}
$$

where $\Delta \mathbf{R}_{j}$ is the ionic displacement in region II during the relaxation, and the last term in the equation represents the work done by the correction force. With the ghost force correction, the coupling errors on region II ions are significantly reduced, as shown in Table I, by comparing the results between system No. 1 and No. 3 .

\section{B. Kohn-Sham density functional theory for quantum mechanics region}

For many material systems, KS-DFT is more desirable than OFDFT for QM calculations of region I despite much less computational expense offered by OFDFT. KS-DFT is superior to OFDFT in several aspects: (1) the kinetic energy is calculated more accurately by KS-DFT; (2) the external potential energy can be calculated more accurately by $\mathrm{KS}$ DFT by using nonlocal pseudopotentials; (3) KS-DFT allows standard electronic structure analysis to be carried out, thanks to the access of KS orbitals. Because of these advantages, we have also implemented KS-DFT in region I for QM calculations. In the following, we demonstrate how a plane-wave pseudopotential KS-DFT approach can be adapted to perform QM/MM calculations. To set the stage for the discussion, we first review the key ingredients of the KS-DFT approach as implemented in VASP. ${ }^{32-34}$ The KS total energy functional is written as ${ }^{32-34}$

$$
\begin{aligned}
E_{\mathrm{KS}}[\{\phi\} ;\{\mathbf{R}\}]= & \sum_{n} f_{n}\left\langle\phi_{n}\left|T+V_{\mathrm{NL}}^{\mathrm{ion}}\right| \phi_{n}\right\rangle+E_{\mathrm{H}}[\rho]+E_{\mathrm{xc}}[\rho] \\
& +\int d \mathbf{r} V_{\mathrm{loc}}^{\text {ion }}(\mathbf{r}) \rho(\mathbf{r})+\gamma_{\text {Ewald }}(\{\mathbf{R}\}),
\end{aligned}
$$

where $n$ is the index of KS orbitals $\phi_{n}$ and $f_{n}$ is the occupation number. $\{\mathbf{R}\}$ denotes the position of the ions. $k$ index is dropped for simplicity. $E_{\mathrm{H}}$ and $E_{\mathrm{xc}}$ is the Hartree energy and the exchange-correlation energy, respectively. $V_{\text {loc }}^{\text {ion }}$ and $V_{\mathrm{NL}}^{\mathrm{ion}}$ is the local and nonlocal parts of the ionic pseudopotential, respectively, and $\gamma_{\text {Ewald }}$ is the Madelung energy. The variation of the KS energy functional with respect to KS orbitals leads to the generalized eigenvalue equations,

$$
\mathbf{H}\left|\phi_{n}\right\rangle=\epsilon_{n} \mathbf{S}\left|\phi_{n}\right\rangle .
$$

Here, $\mathbf{S}$ is the overlap matrix, and $\mathbf{H}$ is the KS Hamiltonian given as

$$
\mathbf{H}=T+V_{\mathrm{loc}}+V_{\mathrm{NL}} .
$$

The local potential is given by $V_{\mathrm{loc}}=V_{\mathrm{loc}}^{\text {ion }}+V_{\mathrm{H}}[\rho]+V_{\mathrm{xc}}[\rho]$, with $V_{\mathrm{H}}[\rho], V_{\mathrm{xc}}[\rho]$, and $V_{\mathrm{NL}}$ being the Hartree potential, exchange-correlation potential, and nonlocal potential, respectively. For a given charge density $\rho$, one can determine the band-structure energy $\epsilon_{n}$ and the KS orbitals $\phi_{n}$ from the $\mathrm{KS}$ eigenvalue equations. The total energy is thus calculated as

$$
E_{\mathrm{tot}}=\sum_{n} f_{n} \epsilon_{n}+E_{\mathrm{d} . \mathrm{c} .}[\rho]+\gamma_{\mathrm{Ewald}},
$$

with the double counting energy term,

$$
E_{\mathrm{d} . \mathrm{c} .}[\rho]=-E_{\mathrm{H}}[\rho]+E_{\mathrm{xc}}[\rho]-\int d \mathbf{r} V_{\mathrm{xc}}(\mathbf{r}) \rho(\mathbf{r})
$$

The quantum coupling of QM/MM is achieved by minimizing the sum, $\left\{E_{\mathrm{KS}}\left[\rho^{\mathrm{I}} ; \mathbf{R}^{\mathrm{I}}\right]+E_{\mathrm{OF}}^{\mathrm{int}}\left[\rho^{\mathrm{I}}, \rho^{\mathrm{II}} ; \mathbf{R}^{\mathrm{I}}, \mathbf{R}^{\mathrm{II}}\right]\right\}$ with respect to $\rho^{\mathrm{I}}$, as shown in Eq. (1). The variation of the sum leads to an additional potential term (embedding potential) in the KS Hamiltonian, and now, the local potential in Eq. (28) becomes

$$
V_{\mathrm{loc}}=V_{\mathrm{loc}}^{\mathrm{ion}}+V_{\mathrm{H}}\left[\rho^{\mathrm{I}}\right]+V_{\mathrm{xc}}\left[\rho^{\mathrm{I}}\right]+\mu_{\mathrm{emb}}\left[\rho^{\mathrm{I}}, \rho^{\mathrm{II}}\right] .
$$

The double counting term in the total energy expression is also modified accordingly, 


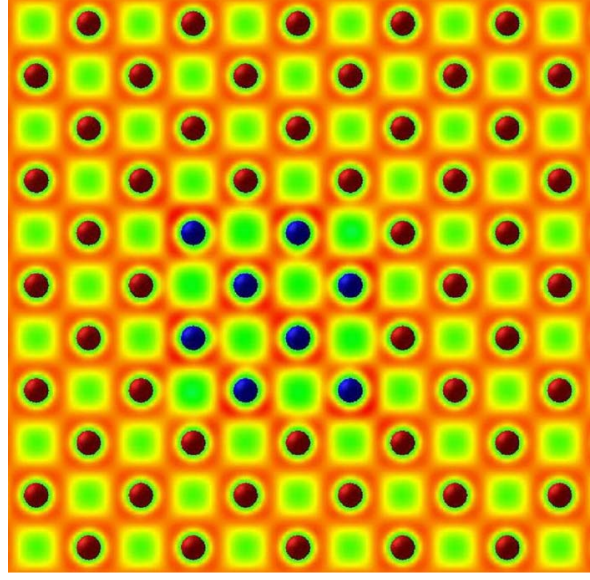

FIG. 5. (Color online) Total charge density $\rho^{\text {tot }}$ (in $\AA^{-3}$ ) for $\mathrm{Al}$ perfect lattice with KS-DFT for region I. Contour scale ranges from 0.0031 (blue) to 0.0342 (red). Blue and red spheres represent the ions belonging to regions I and II, respectively.

$$
\begin{aligned}
E_{\text {d.c. }}= & -E_{\mathrm{H}}\left[\rho^{\mathrm{I}}\right]+E_{\mathrm{xc}}\left[\rho^{\mathrm{I}}\right]-\int d \mathbf{r} V_{\mathrm{xc}}(\mathbf{r}) \rho^{\mathrm{I}}(\mathbf{r}) \\
& +E_{\mathrm{OF}}^{\mathrm{int}}\left[\rho^{\mathrm{I}}, \rho^{\mathrm{I}} ; \mathbf{R}^{\mathrm{I}}, \mathbf{R}^{\mathrm{I}}\right]-\int d \mathbf{r} \mu_{\mathrm{emb}}(\mathbf{r}) \rho^{\mathrm{I}}(\mathbf{r}) .
\end{aligned}
$$

Following the same numerical schemes as implemented in VASP, $\rho^{\mathrm{I}}$ can be self-consistently determined for a given ionic configuration, and the total energy can be calculated. The calculation of force $-\partial E_{\mathrm{QM}} / \partial \mathbf{R}_{i}^{\mathrm{I}}$ is carried out in the same way as implemented in VASP except an additional term from the embedding potential being added,

$$
\int d \mathbf{r} \frac{\partial \mu_{\mathrm{emb}}}{\partial \rho^{\mathrm{I}}} \frac{\partial \rho_{\mathrm{atom}}^{\mathrm{I}}(\mathbf{r})}{\partial \mathbf{R}^{\mathrm{I}}}\left[\rho_{\text {out }}^{\mathrm{I}}(\mathbf{r})-\rho_{\text {in }}^{\mathrm{I}}(\mathbf{r})\right] .
$$

Here, $\rho_{\text {atom }}^{\mathrm{I}}$ is the superposition of the atomic charge densities in region $\mathrm{I}, \rho_{\text {out }}^{\mathrm{I}}$ is the converged charge density, and $\rho_{\text {in }}^{\mathrm{I}}$ is the charge density obtained from the previous iteration.

Since there are two different energy functionals (KS-DFT and OFDFT) involved in the self-consistent calculation of $\rho^{\mathrm{I}}$, attention must be paid to the different treatments of kinetic energy and pseudopotential in order to ensure a seamless coupling. For the former, one needs to adjust the parameters for the kinetic energy functional in OFDFT to best match the KS-DFT results. For the later, one needs to construct a local pseudopotential that best reproduces the results of the nonlocal pseudopotential used in KS-DFT. In the present work, we use the same local pseudopotential as in VASP for a radial distance beyond the core cutoff, but with a repulsive local potential developed by Goodwin et al. ${ }^{31}$ elsewhere. The local pseudopotential is constructed to minimize $\delta E_{\mathrm{OF}}^{\mathrm{tot}}[\rho] / \delta \rho$ with $\rho(\mathbf{r})$ calculated by KS-DFT for a unit cell of a perfect lattice.

A plane-wave cutoff of $300 \mathrm{eV}$ and a Monkhorst-Pack $k$ mesh of $1 \times 1 \times 9$ (Ref. 41) are used for test system No. 6 in Table I. The contour of total charge density for the test bulk system is presented in Fig. 5, and the forces and atomic displacement of the same system (No. 6) are summarized in
Table I. Although the coupling errors of the second method seem slightly greater than the first one, the advantages of the KS-DFT still render the second method attractive for certain material systems.

\section{APPLICATION: BINDING ENERGY OF VACANCY CLUSTERS IN AL}

Next, we present an application of the first coupling method to calculate the binding energy of vacancy clusters in Al. Although $a b$ initio calculations have been performed for small vacancy clusters in $\mathrm{Al},{ }^{42-45}$ little effort has been devoted to QM calculations of relatively larger vacancy clusters, which are the key for understanding void formation in materials. By using the QM/MM multiscale approach, we are able to carry out large scale calculations consisting of up to ten vacancy in a cluster. First, we benchmark the first coupling method by comparing the binding energy and charge density of a divacancy to the previous $a b$ initio results. We then predict the binding energy of larger vacancy clusters.

At a constant pressure, the formation energy of an $n$-vacancy cluster can be calculated as ${ }^{42-46}$

$$
E_{n \mathrm{v}}^{\mathrm{F}}=E_{n \mathrm{v}}^{\mathrm{tot}}(N-n)-\frac{N-n}{N} E^{\mathrm{tot}}(N),
$$

where $E_{n v}^{\mathrm{tot}}(N-n)$ is the total energy of the supercell containing $N-n$ atoms with an $n$-vacancy cluster and $E^{\text {tot }}(N)$ is the total energy of the supercell containing $N$ atoms in the perfect lattice configuration. The binding energy of an $n$-vacancy cluster is evaluated by

$$
E_{n \mathrm{v}}^{\mathrm{B}}=n E_{1 \mathrm{v}}^{\mathrm{F}}-E_{n \mathrm{v}}^{\mathrm{F}} \text {. }
$$

Here, the first method (OFDFT for region I) is employed to compute the binding energy of vacancy clusters with the size ranging from 2 (divacancy) to 10 . We choose a large supercell of $14 a_{0} \times 14 a_{0} \times 14 a_{0}$ for the calculations; it con-

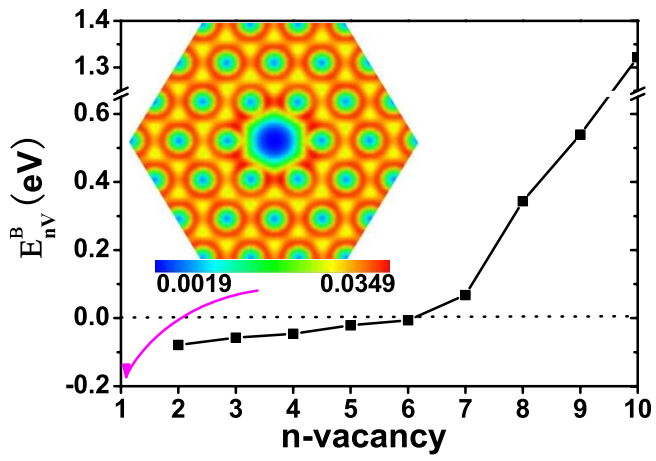

FIG. 6. (Color online) Binding energy (eV) of $n$-vacancy clusters versus number $n$. The negative (positive) value of $E_{n v}^{\mathrm{B}}$ indicates the $n$-vacancy cluster is energetically unstable (stable) against the simultaneous dissociation into $n$ monovacancies. Inset: electron density $\left(\AA^{-3}\right)$ in a close-packed $\{111\}$ plane in the presence of a monovacancy. The vacancy induces an accumulation of charge density (a red ring) between the atoms in the first shell around the vacancy. 
tains $N=10976$ atoms and is partitioned into region I (256 atoms) and region II (10720 atoms). The results of binding energy are shown in Fig. 6.

The binding energy of a divacancy in $\mathrm{Al}$ from the first coupling method is $-0.08 \mathrm{eV}$ which is identical to the value obtained from a stand-alone KS-DFT calculation. ${ }^{42-44}$ In addition, we find that there is a charge accumulation (or a bonding ring) formed between the atoms in the first shell around the vacancy (see the inset of Fig. 6). This vacancyinduced circular bonding has also been observed by others from stand-alone KS-DFT calculations. ${ }^{42,44}$ The comparisons here suggest that the present $\mathrm{QM} / \mathrm{MM}$ method reproduces the stand-alone KS-DFT results, a strong indication of the success of the coupling method. We have recently applied the method to study the electronic origin of void formation in $\mathrm{Al}^{47}$

\section{CONCLUSION}

We have presented a self-consistent quantum coupling scheme that combines DFT and empirical atomistic simulations concurrently. The coupling formalism is based on OFDFT and represents a quantum mechanical formulation of
QM/MM coupling. Two energy functionals are used for region I: one is based on OFDFT and the other on KS-DFT. The numerical implementations of the coupling methods, including periodic boundary conditions, construction of atomic charge density, and ghost force corrections, are described in detail. We explain how a standard plane-wave pseudopotential KS-DFT code can be adapted to perform the proposed $\mathrm{QM} / \mathrm{MM}$ calculations. We use the perfect lattice of $\mathrm{Al}$ as the test system from which the coupling errors can be evaluated unambiguously. The tests indicate that the present QM/MM schemes give excellent results in terms of coupling. We have also applied the QM/MM method to compute the binding energy of vacancy clusters in $\mathrm{Al}$, which yields exactly the same results for a divacancy compared to stand-alone KSDFT calculations. All these results strongly suggest that the present QM/MM methods are excellent candidates for multiscale simulations of metallic systems.

\section{ACKNOWLEDGMENTS}

This work was supported in part by NSF PREM under Grant No. DMR-0611562, DOE SciDAC under Grant No. DE-FC02-06ER25791, and ACS under Grant No. PRF 43993-AC10. *ganglu@csun.edu

${ }^{1}$ G. Lu and E. Kaxiras, in Handbook of Theoretical and Computational Nanotechnology, edited by M. Rieth and W. Schommers (American Scientific, Stevenson Ranch, CA, 2004), Chap. 22.

${ }^{2}$ H. Lin and D. G. Truhlar, Theor. Chem. Acc. 117, 185 (2007).

${ }^{3}$ F. F. Abraham, R. Walkup, H. Gao, M. Duchaineau, T. de la Rubia, and M. Seager, Proc. Natl. Acad. Sci. U.S.A. 99, 5777 (2002).

${ }^{4}$ E. B. Tadmor, M. Ortiz, and R. Phillips, Philos. Mag. A 73, 1529 (1996).

${ }^{5}$ J. Gao, in Reviews in Computational Chemistry, edited by K. B. Libkowitz and D. B. Boyd (VCH, New York, 1996), pp. 119185.

${ }^{6}$ J. Q. Broughton, F. F. Abraham, N. Bernstein, and E. Kaxiras, Phys. Rev. B 60, 2391 (1999).

${ }^{7}$ N. Bernstein and D. Hess, Multiscale Modelling of Materials, edited by L. P. Kubin, J. L. Bassani, K. Cho, H. Gao, and R. L. B. Selinger, MRS Symposia Proceedings No. 653 (Materials Research Society, Pittsburgh, 2001), p. Z2.7.1.

${ }^{8}$ E. Lidorikis, M. E. Bachlechner, R. K. Kalia, A. Nakano, P. Vashishta, and G. Z. Voyiadjis, Phys. Rev. Lett. 87, 086104 (2001).

${ }^{9}$ V. B. Shenoy, R. Miller, E. B. Tadmor, D. Rodney, R. Phillips, and M. Ortiz, J. Mech. Phys. Solids 47, 611 (1999).

${ }^{10}$ N. Govind, Y. A. Wang, A. J. R. da Silva, and E. A. Carter, Chem. Phys. Lett. 295, 129 (1998).

${ }^{11}$ N. Choly, G. Lu, Weinan E., and E. Kaxiras, Phys. Rev. B 71, 094101 (2005).

${ }^{12}$ G. Lu, E. B. Tadmor, and E. Kaxiras, Phys. Rev. B 73, 024108 (2006).

${ }^{13}$ C. Y. Wang and X. Zhang, Curr. Opin. Solid State Mater. Sci. 10, 2 (2006).
${ }^{14}$ T. Mordasini and W. Thiel, Chimia 52, 288 (1998).

${ }^{15}$ T. K. Woo, P. M. Margl, L. Deng, L. Cavallo, and T. Ziegler, ACS Symp. Ser. 721, 173 (1999).

${ }^{16} \mathrm{P}$. Sherwood, in Modern Methods and Algorithms of Quantum Chemistry, edited by J. Grotendorst (NIC, Princeton, 2000), vol. 3, p. 285.

${ }^{17}$ J. Gao and D. G. Truhlar, Annu. Rev. Phys. Chem. 53, 467 (2002).

${ }^{18}$ X. Li and W. E., J. Mech. Phys. Solids 53, 1650 (2005).

${ }^{19}$ P. Huang and E. A. Carter, J. Chem. Phys. 125, 084102 (2006).

${ }^{20}$ P. Garcia-Gonzalez, J. E. Alvarellos, and E. Chacon, Phys. Rev. B 53, 9509 (1996).

${ }^{21}$ L. W. Wang and M. P. Teter, Phys. Rev. B 45, 13196 (1992).

${ }^{22}$ Y. A. Wang, N. Govind, and E. A. Carter, Phys. Rev. B 60, 16350 (1999).

${ }^{23}$ W. Kohn and L. Sham, Phys. Rev. 140, A1133 (1965).

${ }^{24}$ M. S. Daw and M. I. Baskes, Phys. Rev. B 29, 6443 (1984).

${ }^{25}$ N. Choly and E. Kaxiras, Solid State Commun. 121, 281 (2002).

${ }^{26}$ L. H. Thomas, Proc. Cambridge Philos. Soc. 23, 542 (1926).

${ }^{27}$ E. Fermi, Z. Phys. 48, 73 (1928).

${ }^{28}$ C. F. von Weizsacker, Z. Phys. 96, 431 (1935).

${ }^{29}$ J. Lindhard, K. Dan. Vidensk. Selsk. Mat. Fys. Medd. 28, 8 (1954).

${ }^{30}$ J. P. Perdew, and A. Zunger, Phys. Rev. B 23, 5048 (1981).

${ }^{31}$ L. Goodwin, R. J. Needs, and V. Heine, J. Phys.: Condens. Matter 2, 351 (1990).

${ }^{32}$ G. Kresse and J. Hafner, Phys. Rev. B 47, 558 (1993); 49, 14251 (1994).

${ }^{33}$ G. Kresse and J. Furthmuller, Phys. Rev. B 54, 11169 (1996).

${ }^{34}$ G. Kresse and J. Furthmuller, Comput. Mater. Sci. 6, 15 (1996).

${ }^{35}$ F. Ercolessi, M. Parrinello, and E. Tosatti, Philos. Mag. A 58, 213 
(1988).

${ }^{36}$ F. Ercolessi and J. Adams, Europhys. Lett. 26, 583 (1994).

${ }^{37}$ C. Herring, Phys. Rev. A 34, 2614 (1986).

${ }^{38}$ E. Clementi and C. Rotti, Atomic Data and Nuclear Data Tables (Academic, New York, 1974), Vol. 14, pp. 3 and 4.

${ }^{39}$ G. H. Wannier, Phys. Rev. 52, 191 (1937).

${ }^{40}$ N. Marzari and D. Vanderbilt, Phys. Rev. B 56, 12847 (1997).

${ }^{41}$ H. J. Monkhorst and J. D. Pack, Phys. Rev. B 13, 5188 (1976).

${ }^{42}$ K. Carling, G. Wahnstrom, T. R. Mattsson, A. E. Mattsson, N Sandberg, and G. Grimvall, Phys. Rev. Lett. 85, 3862 (2000).
${ }^{43}$ K. M. Carling, G. Wahnstrom, T. R. Mattsson, N. Sandberg, and G. Grimvall, Phys. Rev. B 67, 054101 (2003).

${ }^{44}$ T. Uesugi, M. Kohyama, and K. Higashi, Phys. Rev. B 68, 184103 (2003).

${ }^{45}$ P. A. Korzhavyi, I. A. Abrikosov, B. Johansson, A. V. Ruban, and H. L. Skriver, Phys. Rev. B 59, 11693 (1999).

${ }^{46}$ Y. Mishin, M. R. Sorensen, and A. F. Voter, Philos. Mag. A 81, 2591 (2001).

${ }^{47}$ X. Zhang and G. Lu (unpublished). 